\title{
CPT1a maintains phenotype of tubules via mitochondrial respiration during kidney injury and repair
}

\author{
Qi Yuan ${ }^{1,2,3}$, Yunhui $\mathrm{Lv}^{1,3}$, Hao Ding ${ }^{1}$, Qingqing Ke ${ }^{1}$, Caifeng $\mathrm{Shi}^{1}$, Jing Luo ${ }^{1}$, Lei Jiang $\mathbb{D}^{1 凶}$, Junwei Yang $\mathbb{D}^{1 凶}$ and Yang Zhou $\mathbb{D}^{1 凶}$
}

(c) The Author(s) 2021

Impaired energy metabolism in proximal tubular epithelial cells (PTECs) is strongly associated with various kidney diseases. Here, we characterized proximal tubular phenotype alternations during kidney injury and repair in a mouse model of folic acid nephropathy, in parallel, identified carnitine palmitoyltransferase 1a (CPT1a) as an energy stress response accompanied by renal tubular dedifferentiation. Genetic ablation of Cpt1a aggravated the tubular injury and interstitial fibrosis and hampered kidney repair indicate that CPT1a is vital for the preservation and recovery of tubular phenotype. Our data showed that the lipid accumulation and mitochondrial mass reduction induced by folic acid were persistent and became progressively more severe in PTECs without CPT1a. Interference of CPT1a reduced capacities of mitochondrial respiration and ATP production in PTECs, and further sensitized cells to folic acid-induced phenotypic changes. On the contrary, overexpression of CPT1a protected mitochondrial respiration and prevented against folic acid-induced tubular cell damage. These findings link CPT1a to intrinsic mechanisms regulating the mitochondrial respiration and phenotype of kidney tubules that may contribute to renal pathology during injury and repair.

Cell Death and Disease (2021)12:792; https://doi.org/10.1038/s41419-021-04085-w

\section{INTRODUCTION}

The kidney requires a large amount of ATP to remove waste and reabsorb nutrients, to modulate the balance of electrolytes, fluid, and acid-base homeostasis [1]. Energy metabolism in kidney proximal tubular epithelial cells (PTECs) is particularly unique because ATP production here primarily depends on oxidative phosphorylation (OXPHOS) of fatty acid in mitochondria which is more efficient and safe in energy generation by oxidation of glucose [2, 3]. Evidence has accumulated to show that PTECs are not uninvolved in the process of kidney injury. They undergo phenotypic changes, acquire mesenchymal functions, and hence contribute to the synthesis of extracellular matrix proteins $[4,5]$. The high-energy requirement and the significance of energy metabolism in tubular cells have been appreciated for many years; however, a strong link between impaired metabolic homeostasis and renal injury has only emerged recently [6].

After kidney injury, the PTECs die or undergo dedifferentiation and proliferation to repair [7]. Redifferentiation of the reconstituted epithelium is the major pathway towards the recovery of normal kidney structure and function. However, this is merely an ideal condition because the redifferentiation of proliferative PTECs varies [8]. Those PTECs failed to redifferentiate become atrophy [9] and trigger tubulointerstitial fibrosis [10]. Although the mechanism by which the PTECs become redifferentiation or atrophy is unknown, the energy metabolism is probably involved. Metabolism alternations occur immediately after injury, including diminished fatty acid oxidation (FAO) [6], activated glycolysis [11], increased lactate release [12], and elevated pyruvate kinase in the kidney [13]. These physiologic changes in regenerating epithelium may become pathologic if persistent. The previous study has suggested that tubules with a glycolytic shift and mitochondrial pathology in the early stage of regeneration after kidney injury probably fail to redifferentiate and progress to atrophy [9].

Transcriptome analysis demonstrated a markedly decreased expression of key enzymes and regulators of FAO in kidney diseases [6]. Recently, through unbiased cell trajectory analyses, it revealed that differentiation of PTECs was altered in kidney disease, which was strongest and most reproducible associated with OXPHOS and FAO in tubules [14]. The first and rate-limiting component of FAO system is carnitine palmitoyltransferase 1 (CPT1). Several studies indicated that CPT1 protects against kidney disease [6, 15-18].

On the basis of this, we hypothesized that CPT1a is involved in the mechanism of the maintenance of PTECs phenotype and that its dysregulation is related to the development of tubular damage, poor repair, and subsequent fibrosis. To address these hypotheses, we evaluated renal lipid and the tubular phenotype of inducible PTEC-specific Cpt1a-deficient mice exposed to a folic acid-induced mouse model of kidney injury and repair.

\section{MATERIAL AND METHODS \\ Animal model}

Male C57BL/6J mice purchased from Shanghai experimental animal center were housed in the animal facilities at Nanjing Medical University and were

\footnotetext{
${ }^{1}$ Center for Kidney Disease, Second Affiliated Hospital of Nanjing Medical University, Nanjing, China. ${ }^{2}$ Department of Nephrology, The Affiliated Suzhou Hospital of Nanjing Medical University, Suzhou Municipal Hospital, Gusu School, Nanjing Medical University, Suzhou, China. ${ }^{3}$ These authors contributed equally: Qi Yuan, Yunhui Lv. 凶email: jianglei@njmu.edu.cn; jwyang@njmu.edu.cn; zhouyang@njmu.edu.cn Edited by: Professor Alessandro Finazzi-Agrò
} 
treated humanely according to guidelines of the Institutional Animal Use and Care Committee with free access to water and food. A conditional system was used to knockout CPT1a in the renal tubule of adult mice. Three transgenic mouse lines were cross-bred: Pax8-reverse tetracyclinedependent transactivator (Pax8-rtTA) mice (Jackson lab, stock No: 007176),

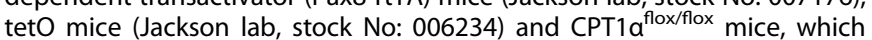
were in-housed generated, with exon 3 of the mouse Cpt1a gene floxed. Tail DNA from all mice was genotyped by PCR analysis. Primers used for CPT1a flox/flox genotyping were as follows: Primer F 5'-GCA GCC CAG CTG ATG ACC TGA G-3'; Primer wild-type R 5'-CCT CTG CCA CTC TTA GCC TAG TC-3'; Primer neo R 5'-TGC TAA AGC GCA TGC TCC AGA C-3'. Doxycyclinecontaining chow was started at 3 weeks of age. Male mice aged 6 -8 weeks were randomly assigned into different groups with at least six mice per group: control, 2 weeks, 4 weeks, and 12 weeks after folic acid. Control mice were injected intraperitoneally with $\mathrm{NaHCO}_{3}(300 \mathrm{mmol} / \mathrm{L})$. Folic acid (F7876, Sigma-Aldrich) was dissolved in $\mathrm{NaHCO}_{3}(300 \mathrm{mmol} / \mathrm{L})$ and injected intraperitoneally at the dose of $250 \mathrm{mg} / \mathrm{kg}[6,19]$. No blinding was done.

\section{Cell culture and treatment}

Primary PTECs were cultured from collagenase-digested cortical fragments of mice (about 21 days) kidneys according to a modified method previously described [20,21]. In brief, after been dissected and collagenase digested, two nylon sieves with the pore sizes of 250 and $80 \mu \mathrm{m}$ were used to yield proximal tubule fragments, which were then washed, resuspended, and seeded onto collagen-coated permeable PTFE-filter supports and cultured for $48 \mathrm{~h}$ in a standard humidified incubator with the medium replaced every 2 days till the organization of a confluent monolayer of cells. At $80 \%$ confluence, cells were starved overnight and treated with $10 \mathrm{mmol} / \mathrm{L}$ folic acid (FA) $[22,23]$. PTECs were transiently transfected with CPT1a siRNA or corresponding negative control (Integrated Biotech Solutions Co., Ltd, Shanghai, China) by lipofectamine RNAiMAX transfection reagent (13778, Invitrogen). CPT1a plasmid (pCPT1a) and control plasmid (pcDNA3) were transfected at $4 \mu \mathrm{g} / \mathrm{ml}$ by lipofectamine 3000 reagent ( $L 3000$, Invitrogen). Twenty-four hours later, PTECs were then exposed to FA. The siRNA sequences of CPT1a and negative control were as follows: CPT1a: sense 5'- GGA GGA GGU AAG ACU ACU AUG-3'; antisense 5'- UAG UAG UCU UAC CUC CUC CUU-3'. Negative control (N.C.): sense 5'-UUC UCC GAA CGU GUC ACG UTT-3'; anti-sense 5'-ACG UGA CAC GUU CGG AGA ATT-3'.

\section{Renal function assay}

Blood urea nitrogen (BUN) and serum creatinine were measured by QuantiChrom Urea and Creatinine Assay kit (DIUR-500, DICT-500, Hayward, $(\mathrm{A})$, respectively.

\section{Morphology assay}

Kidney samples were fixed in $10 \%$ of neutraformalin in $4{ }^{\circ} \mathrm{C}$ overnight followed by paraffin-embedded and sectioned ( $3 \mu \mathrm{m}$ in thickness) for Masson trichrome or Sirius red staining. Slides were viewed with a Nikon Eclipse 80i microscope (DS-Ri1, Nikon, Shanghai, China). The fibrotic area (\%) for each section was calculated using Image-Pro Plus 6.0 software. Ten randomly chosen fields were evaluated for each mouse, and an average score was calculated.

\section{Transmission electron microscopy}

Kidney sections were fixed in glutaraldehyde (3.7\%) followed by osmium tetroxide $(1 \%)$ and were embedded in gelatin $(10 \%)$ to cut into several blocks $\left(<1 \mathrm{~mm}^{3}\right)$. After dehydration and infiltration in increasing concentrations of alcohol and Quetol-812 epoxy resin mixed with propylene oxide, respectively, samples were embedded in fresh Quetol812 epoxy resin and polymerized. Sections were cut into $100 \mathrm{~nm}$ in thickness and post-stained with uranyl acetate $(10 \mathrm{~min})$ and lead citrate ( $5 \mathrm{~min}$ ) and finally observed in a FEI Tecnai T20 TEM (Thermo Fisher Scientific, Carlsbad, CA, USA), operated at $120 \mathrm{kV}$. The number of mitochondria per field $\left(N_{V}, \mathrm{n} / \mu \mathrm{m} 3\right)$ was estimated as previously described [24]. Briefly, mitochondrial profile area density $\left(N_{A}\right)$ was the ratio between the mitochondrial number and proximal tubular area. Mitochondrial volume density $\left(V_{\mathrm{v}}\right)$ was the ratio of grid points falling over mitochondria and the total number of points of the grid container in the proximal tubule. $N_{\mathrm{V}}=(1 / \beta)\left(N_{\mathrm{A}}^{3 / 2} / V_{\mathrm{V}}{ }^{1 / 2}\right)$, where $\beta$ is calculated using the ratio of the harmonic mean of major and minor axes of mitochondrial sections measured on digital images.

\section{Mitochondrial DNA copy number determination}

Genomic DNA was extracted using the DNeasy Blood \& Tissue Kit (69504, Qiagen). The abundance of mitochondrial DNA (mtDNA) was measured using Mouse mtDNA Copy Number Assay Kit (MCN3, Detroit R\&D). Relative mtDNA copy number was the ratio of mtDNA to nuclear DNA.

\section{Western immunoblot analysis}

Western blot was performed using a lysate of kidney cortex or cultured PTECs. The primary antibodies used were as follows: anti-CPT1a (ab128568, Abcam), anti-KIM1 (ab47635, Abcam), anti-NGAL (ab63929, Abcam), anti-Ecadherin (610181, BD Company), anti-AQP1 (ab168387, Abcam), antivimentin (ab92547, Abcam), anti-fibronectin (F3648, Sigma Aldrich), anticollagen I (1310-01, Southern Biotech) and anti-Tubulin (T6074, Sigma Aldrich). Western blot was performed three times independently. Quantification was completed by scanning and analyzing the intensity of hybridization signals by using NIH Image program.

\section{Immunohistochemical staining}

Paraffin-embedded kidney sections were applied to immunohistochemical staining. They were stained with CPT1a antibody (ab128568, Abcam), KIM1 antibody (SAB3500252, Merck), E-cadherin antibody (610181, BD Company), collagen I antibody (1310-01, Southern Biotech) and fibronectin antibody (F3648, Sigma Aldrich) using the Vector Mouse on Mouse (M.O. M.) immunodetection Kit (Vector Laboratories, Burlingame, CA). Isotype control was also performed.

\section{Immunofluorescent staining}

Kidney tissue cryosection ( $3 \mu \mathrm{m}$ in thickness) was fixed in paraformaldehyde $(4 \%)$, permeabilized with Triton X-100 (0.2\%), blocked with donkey serum (2\%), and then immunostained with antibodies. Similarly, cells were washed, fixed, blocked, and then incubated with specific antibody: E-cadherin antibody (610181, BD Company), anti-AQP1 (AB2219, Millipore), anti-vimentin (sc-6260, Santa), anti-laminin (ab11575 or ab44941, both from Abcam), anti-collagen I (1310-01, Southern Biotech), or antifibronectin (F3648, Sigma Aldrich). Secondary antibodies were FITC or TRITC-conjugated. Cell nuclei were visualized by 40, 6-diamidino-2phenylindole (DAPI) staining. Slides were viewed under a confocal inverted laser microscope (LAM 510 Meta, Zeiss).

\section{Lipid droplets staining}

OCT-embedded kidney tissues were sectioned at $12 \mu \mathrm{m}$ for oil red $\mathrm{O}$ (Sigma-Aldrich, US) and $3 \mu \mathrm{m}$ for bodipy (D3922, Thermo Fisher, US) staining as previously described $[6,21,25]$. Nuclei were viewed by alum haematoxylin staining. After bodipy staining, slides were immunostained with laminin (L9393, Sigma-Aldrich) and DAPI to visualize the tubule and cell nuclei, respectively. The positive area (\%) for each section was analyzed using Image-Pro Plus 6.0 software. At least ten randomly chosen fields were evaluated for each sample, and an average score was calculated.

\section{Measurement of oxygen consumption rate (OCR)}

OCR was measured using a Seahorse Bioscience X24 extracellular flux analyzer (XF24 V7, Seahorse Bioscience). PTECs were seeded in XF24 V7 cell culture microplate at a $1.0 \times 10^{4}$ cells per well. OCR ( $\left.\mathrm{pmol} / \mathrm{min}\right)$ was assessed at baseline and after the addition of $1 \mu \mathrm{mol} / \mathrm{L}$ of oligomycin, followed by $0.75 \mu \mathrm{mol} / \mathrm{L}$ of carbonyl cyanide 4-(trifluoromethoxy) phenylhydrazone (FCCP), and finally $1 \mu \mathrm{mol} / \mathrm{L}$ of oligomycin and rotenone. Protein concentration was measured for normalization.

\section{Statistical analysis}

Statistical analysis of data was performed using Sigma Stat software (Jandel Scientific Software, San Rafael, CA). Data were expressed as mean $\pm \mathrm{SD}$. Comparisons between groups were made using one-way ANOVA, followed by the $t$ test. $P<0.05$ was considered significant.

\section{RESULTS \\ Expression of CPT1a during kidney injury and repair in folic acid nephropathy model}

Models of kidney injury and repair were established by folic acid nephropathy $[19,26]$, which was verified by dynamic changes of serum renal function parameters BUN (Fig. 1A) and creatinine (Fig. 
A
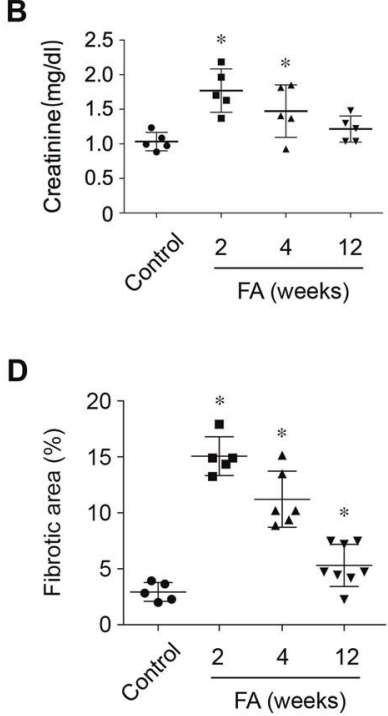

G

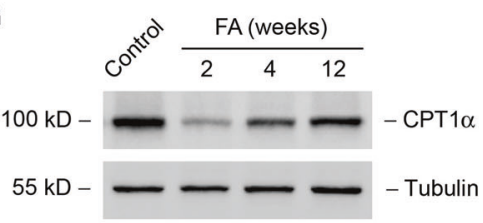

C

E
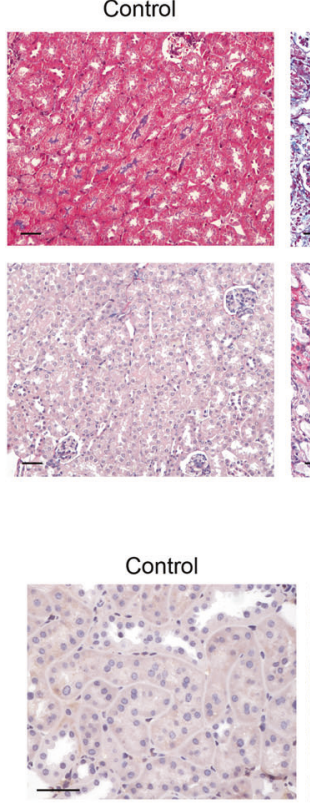

FA 4w
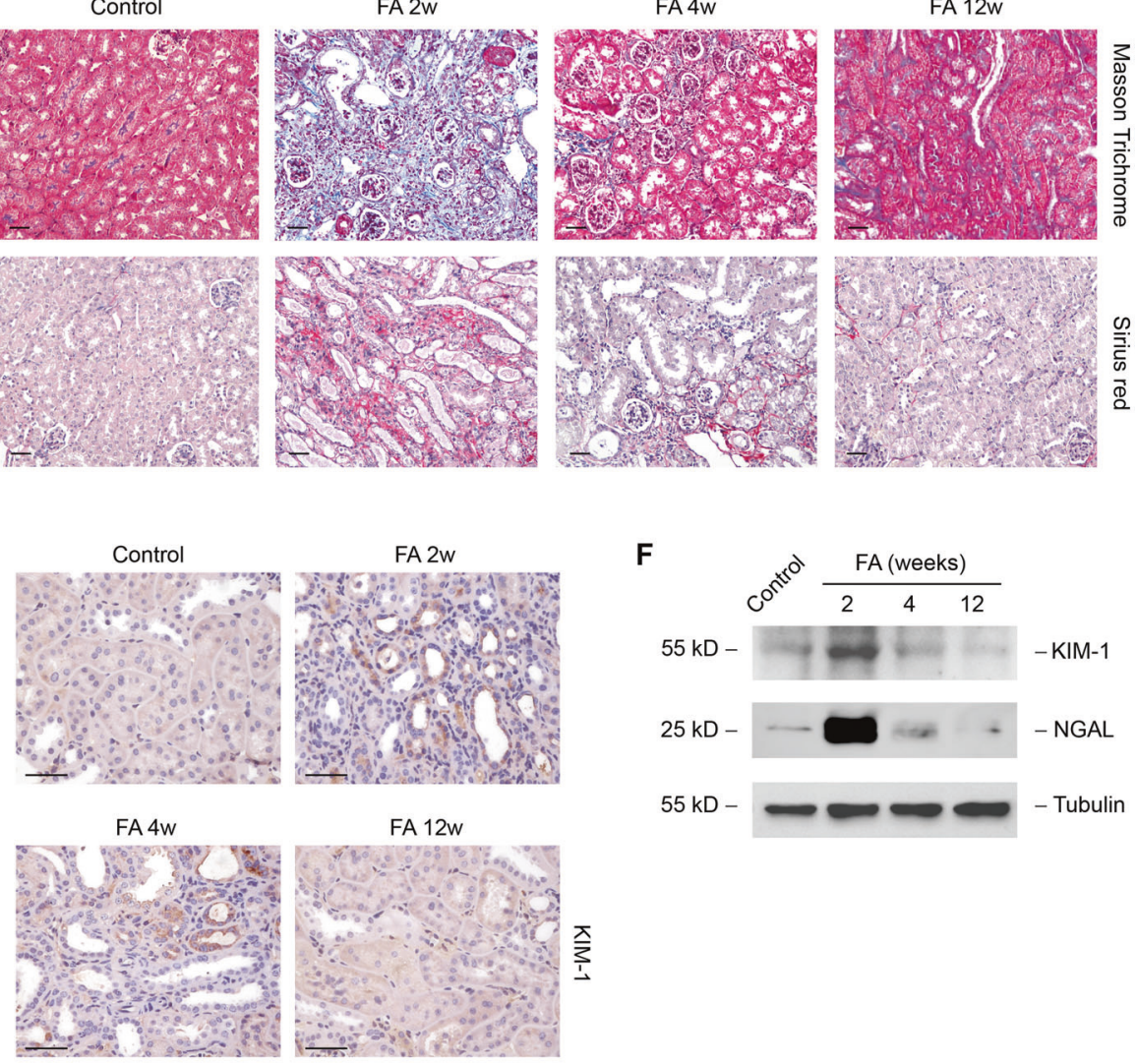

FA $2 w$

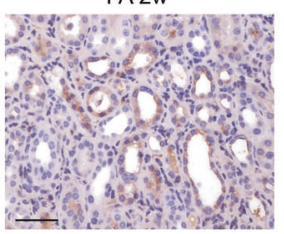

FA 12 w
F

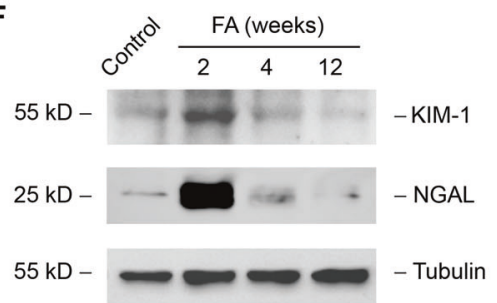

H

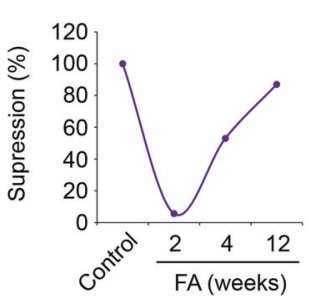

I
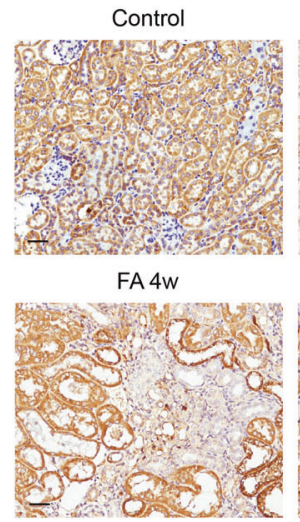

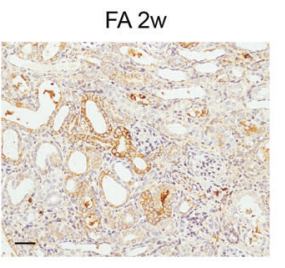

FA $12 w$

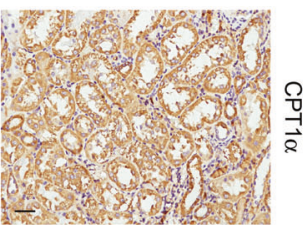

Fig. 1 Dynamic changes of CPT1a during kidney injury and repair in folic acid nephropathy model. Changes of BUN (A) and creatinine (B) in groups as indicated. $n=5$ for each group. ${ }^{*} P<0.05$ versus control. C Representative images of mice kidney samples from groups as indicated stained with Masson trichrome and Sirius red. D Percentage of the fibrotic area in groups as indicated. $n=5-8$ for each group. * $P<0.05$ versus control. E Representative images of mice kidney samples immunostained with KIM-1. F Western blot analysis of protein expression of KIM-1 and NGAL in kidney samples from groups as indicated. G Western blot analysis of protein expression of CPT1 $\alpha$ in kidney samples from groups as indicated. $\mathbf{H}$ Graphic presentation of the relative abundance of CPT1 $\alpha$ in the folic acid model. I Representative images of mice kidney samples immunostained with CPT1 $\alpha$. Scale bar, $50 \mu \mathrm{m}$.

1B). Consistently, the morphologic analysis showed that the tubulointerstitial fibrosis induced by folic acid at 2 weeks was relieved spontaneously at 12 weeks (Fig. 1C, D). Tubular epithelial cells injury markers KIM-1 and NGAL barely detected in normal kidney were markedly increased at 2 weeks after folic acid and then declined at 12 weeks by immunohistochemical staining (Fig. $1 \mathrm{E})$ and western blotting (Fig. 1F). Notably, expression of CPT1a was decreased and recovered during kidney injury (at 2 weeks) and repair (from 4 weeks till 12 weeks), respectively (Fig. 1G, H). Immunohistochemical staining showed the dynamic expression of CPT1a in tubular epithelial cells in FA model (Fig. 1I).

\section{Changes of tubular cell phenotype in FA model}

To gain further insight into the molecular events underlying tubular cell injury and repair, an analysis of tubular cell phenotype from folic acid mice was performed. Several tubular cells markers including E-cadherin and AQP1 were decreased at 2 weeks after folic acid and recovered at 12 weeks by western blotting (Fig. 2A, B) and immunostaining (Fig. 2C). Meanwhile, an opposite alternation was found in the expression of vimentin. Extracellular proteins such as fibronectin and collagen I were upregulated at 2 weeks after folic acid and eliminated at 12 weeks by western blotting (Fig. 2D, E) and immunostaining (Fig. 2F), which were in 
A

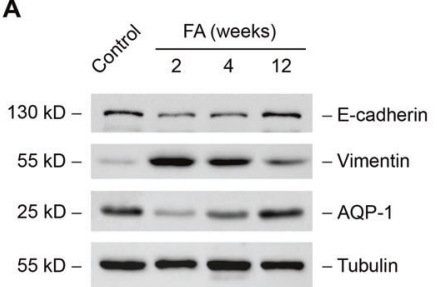

B

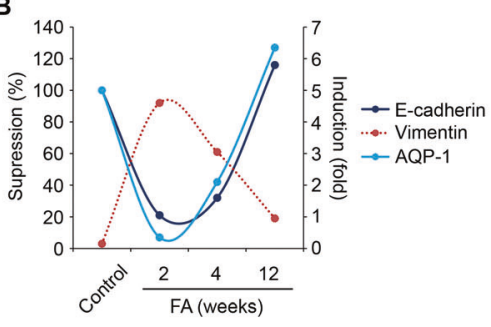

C
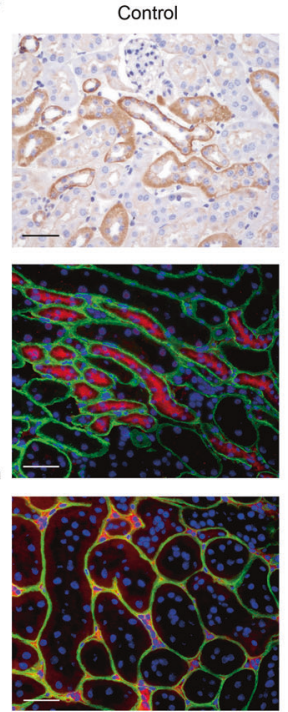

FA $2 w$
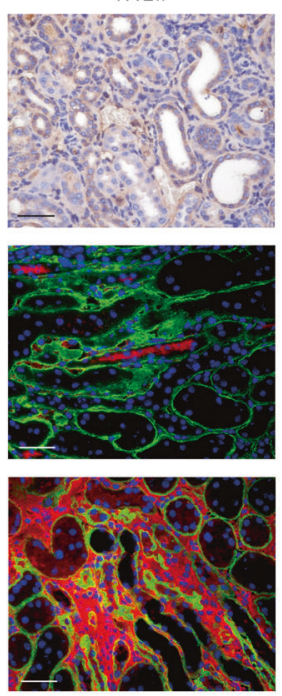
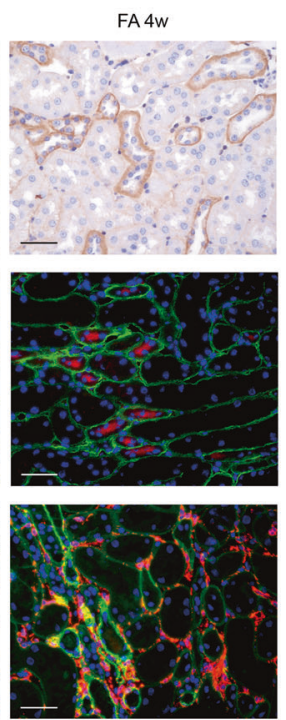
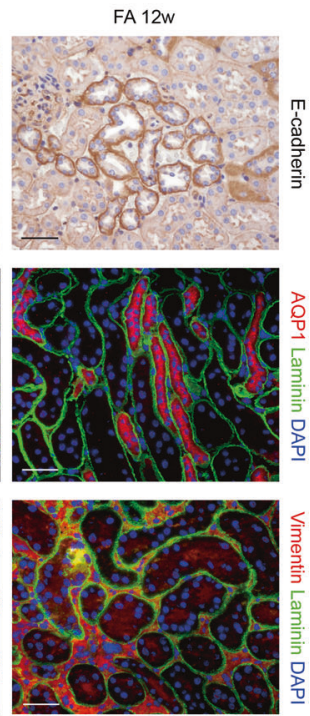

D

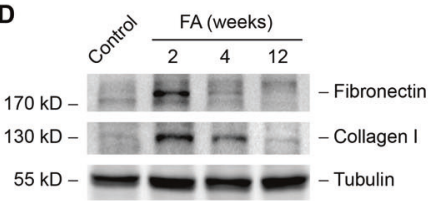

$\mathbf{F}$

E
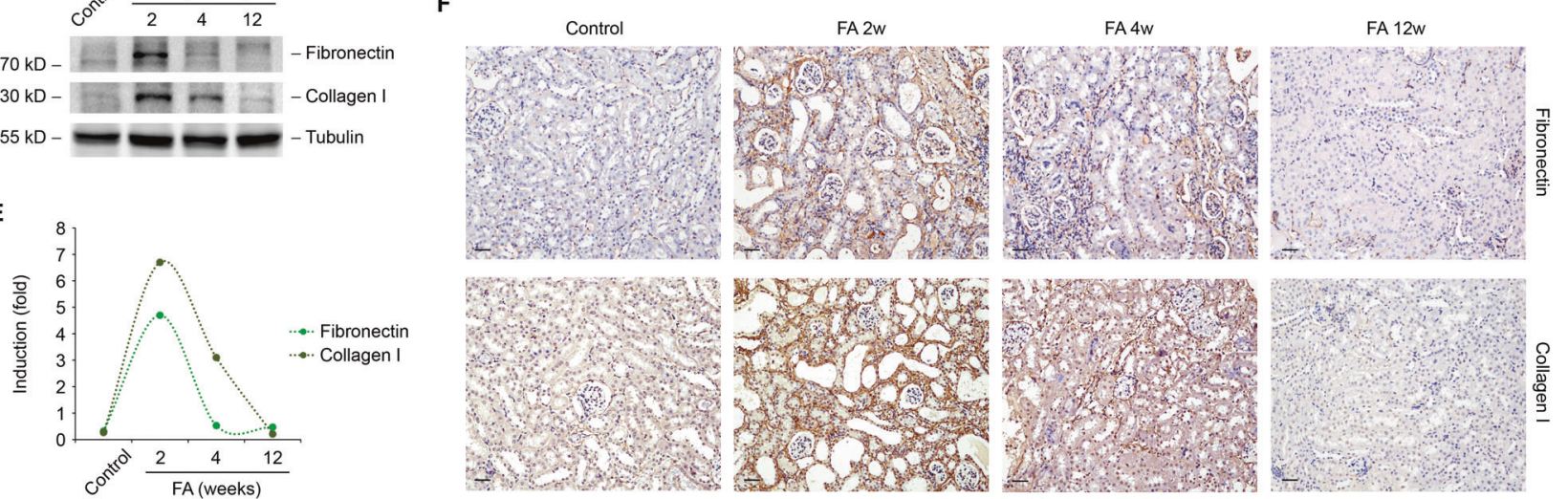

Fig. 2 Changes of tubular cell phenotype in FA model. A Western blot analysis of protein expression of E-cadherin, vimentin, and AQP-1 in kidney samples from groups as indicated. B Graphic presentation of the relative abundance of E-cadherin, vimentin, and AQP-1 in the folic acid model. C Representative images of mice kidney samples immunostained with E-cadherin, AQP-1, and vimentin. D Western blot analysis of protein expression of fibronectin and collagen I in kidney samples from groups as indicated. E Graphic presentation of the relative abundance of fibronectin and collagen I in the folic acid model. F Representative images of mice kidney samples immunostained with fibronectin and collagen I. Scale bar, $50 \mu \mathrm{m}$.

accordance with the dynamic changes of phenotype markers of tubular cell.

\section{Genetic ablation of CPT1a aggravates tubular cell injury and fibrosis and hampers kidney repair}

To further obtain direct evidence that CPT1a within kidney tubule is a direct cause of tubular injury and repair, we generated an inducible, tubular-specific CPT1a knockout mice (CPT1a-/-). Immunoblot analysis of the renal cortex confirmed the ablation of CPT1a in kidney tubule (Fig. 3A). Kidney dysfunction (Fig. 3B, C) and renal fibrosis (Fig. 3D, E) at 2 weeks after folic acid injection were more severe in tubular-specific CPT1a-/- mice. The recovery of kidney function and dissipation of fibrosis were barely absent in CPT1a-/- mice. Genetic ablation of CPT1a resulted in sustained upregulation of KIM-1 and NGAL (Fig. 3F, G) in folic acid mice kidney.

\section{CPT1a is indispensable for preserve and recovery of tubular} phenotype

We further examined the effect of CPT1a deletion on tubular cell phenotype alternation during injury and repair. As compared with CPT1a+/+ mice, the decrease of E-cadherin and AQP1, as well as the increase of vimentin at 2 weeks after folic acid were more significant in CPT1a-/- mice. The altered expression of these phenotype markers persisted at 12 weeks in CPT1a-/- mice by western blotting (Fig. 4A) and immunostaining (Fig. 4B). Similarly, the upregulation (Fig. 4C) and extracellular accumulation (Fig. 4D) of fibronectin and collagen I at 2 weeks after folic acid were more significant in CPT1a-/- mice and do not dissipated at 12 weeks after folic acid.

\section{CPT1a deficiency promotes lipid accumulation and mitochondrial mass reduction}

There was a higher amount of lipid accumulated within the kidney tubules at 2 weeks after folic acid in CPT1a-/- mice compared to $\mathrm{CPT} 1 \mathrm{a}+/+$ mice, which was exemplified by neutral lipid staining and quantification (Fig. 5A, B). The resolution of lipid in tubule of $\mathrm{CPT} 1 \mathrm{a}+/+$ mice at 12 weeks after folic acid was absent in CPT1a $-/-$ mice. Meanwhile, electron micrographs (Fig. 5C) and quantification using morphometric analysis (Fig. 5D) showed that the number of mitochondria in proximal tubules were markedly reduced at 2 weeks in folic acid mice compared with control. Restoration of mitochondria in CPT1a+/+ mice at 12 weeks after folic acid also did not occur in CPT1a-/- mice. As compared with 
A

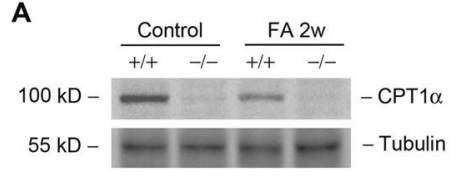

B
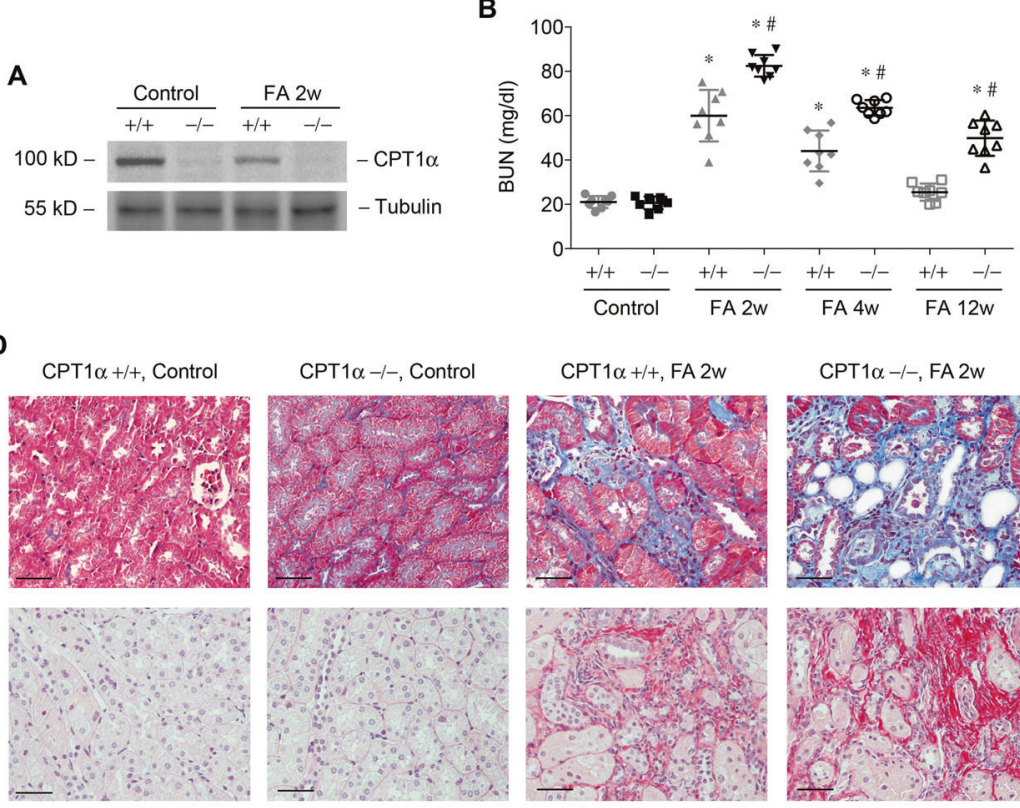

E

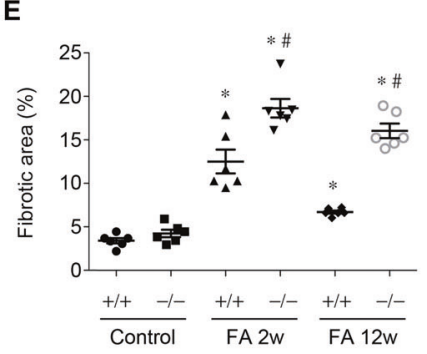

CPT1 $\alpha-/-$, Control
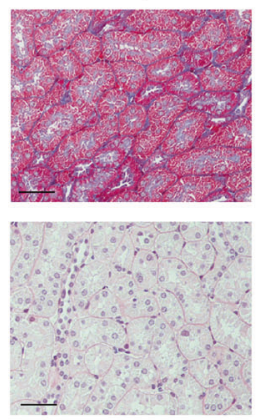

$\mathbf{F}$
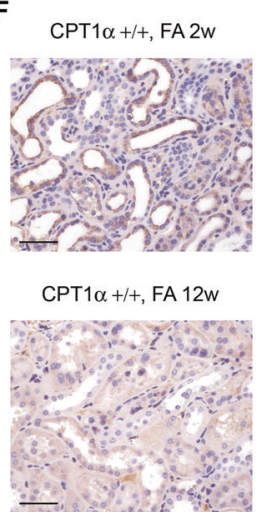

C
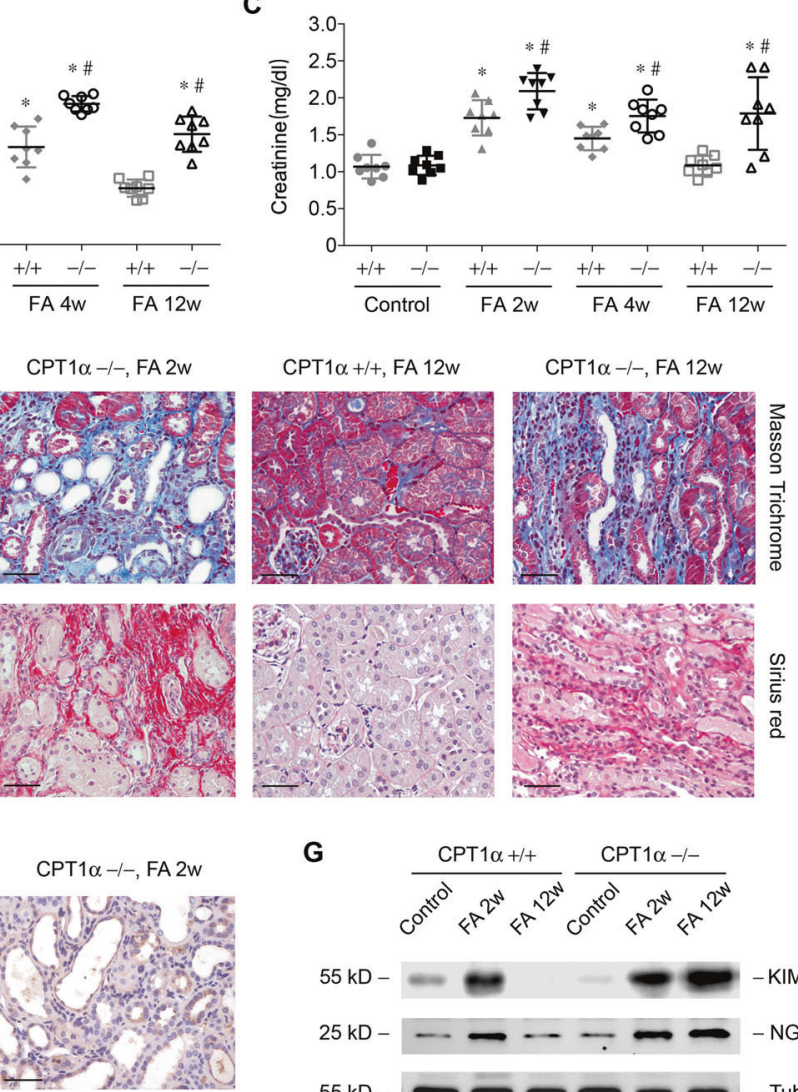

CPT $1 \alpha-/-$, FA $12 w$

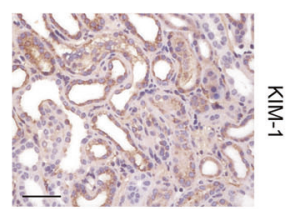

G

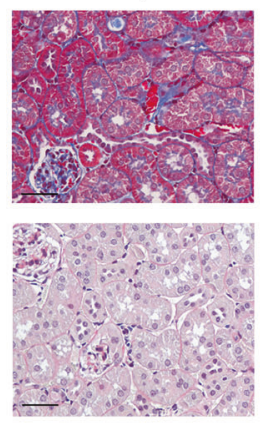

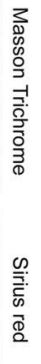
.

CPT $1 \alpha+/+\quad$ CPT $1 \alpha-1-$

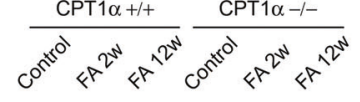

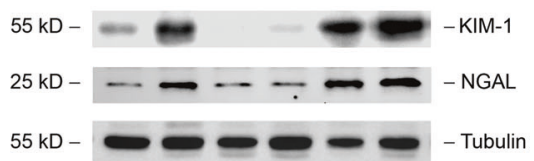

Fig. 3 Genetic ablation of CPT1 a aggravates tubular cell injury and hampers kidney repair. A Western blot analysis of protein expression of CPT1 $\alpha$ in kidney samples from groups as indicated. Changes of BUN (B) and creatinine (C) in groups as indicated. $n=8$ for each group. D Representative images of mice kidney samples from groups as indicated stained with Masson trichrome and Sirius red. E Percentage of the fibrotic area in groups as indicated. $n=6$ for each group. ${ }^{*} P<0.05$ versus control. $\# P<0.05$ versus CPT $1 \alpha+/+$. F Representative images of mice kidney samples immunostained with KIM-1. G Western blot analysis of protein expression of KIM-1 and NGAL in kidney samples from groups as indicated. Scale bar, $50 \mu \mathrm{m}$.

$\mathrm{CPT} 1 \mathrm{a}+/+$ mice, the decrease of mtDNA copy number (Fig. 5E) after folic acid was more significant at 2 weeks and persistent at 12 weeks in CPT1a-/- mice.

\section{CPT1a regulates respiration and ATP production in cultured tubular epithelial cells}

We modulated the expression of CPT1a in primarily cultured tubular epithelial cells by transfection of CPT1a siRNA or plasmid. Immunoblot revealed a markedly decrease in CPT1a protein expression after specific siRNA transfection compared to negative control (N.C.) siRNA (Fig. 6A). Tubular cells with less CPT1a had reduced baseline OCR and a lower ATP production. The FCCPinduced maximal respiration and spare respiration capacity were markedly reduced after the downregulation of CPT1a (Fig. 6B, C). On the contrary, specific plasmid transfection induced a markedly increase in CPT1a protein expression compared to the control plasmid (pcDNA3) (Fig. 6D). In tubular cells with upregulated CPT1a, the baseline OCR and ATP production were elevated remarkably. The FCCP-induced maximal respiration and spare respiration capacity also showed an upward trend after the upregulation of CPT1a (Fig. 6E, F). These results indicate that CPT1a promotes the activity of mitochondrial respiration.

Tubular cells with reduced CPT1a are more sensitive to folic acid-induced alternation

We next provided specific evidence by measuring the effect of CPT1a siRNA on lipid accumulation and tubular cell phenotype in folic acid treatment conditions. Knockdown of CPT1a markedly aggravated the reduction of baseline OCR, maximal respiration, spare respiration capacity, and ATP production (Fig. 7A, B) and resulted in more lipid accumulation (Fig. 7C). Folic acid-induced suppression of tubular markers (E-cadherin and AQP1) and promotion of vimentin and ECM markers (fibronectin and collagen I) were more remarkable by the interference of CPT1a compared to control (Fig. 7D, E).

\section{CPT1a protects mitochondrial respiration and prevents} against folic acid-induced tubular cell damage

We next measured the effect of overexpression of CPT1a on lipid accumulation and tubular cell phenotype in folic acid treatment 

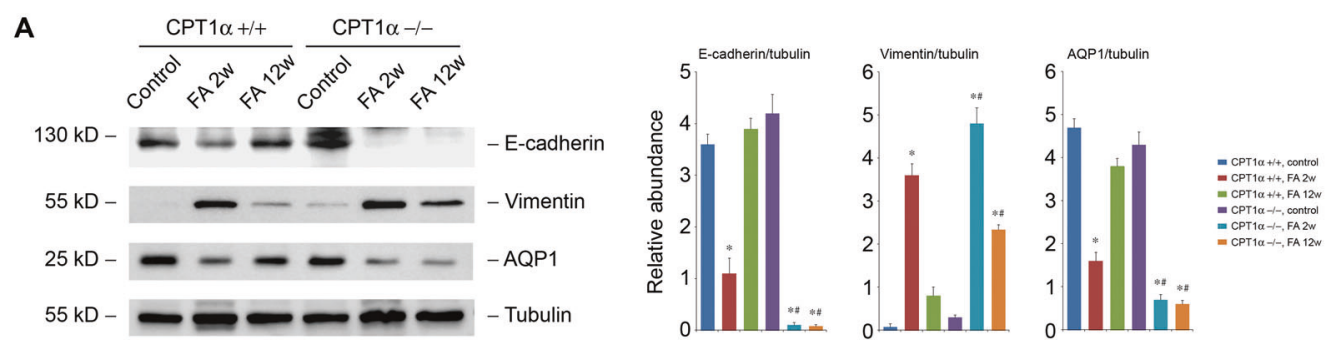

B

CPT $1 \alpha+/+$, FA $2 w$

CPT $1 \alpha-1-$, FA $2 w$

CPT $1 \alpha+/+$, FA $12 w$

CPT1 $\alpha-l-$, FA $12 w$
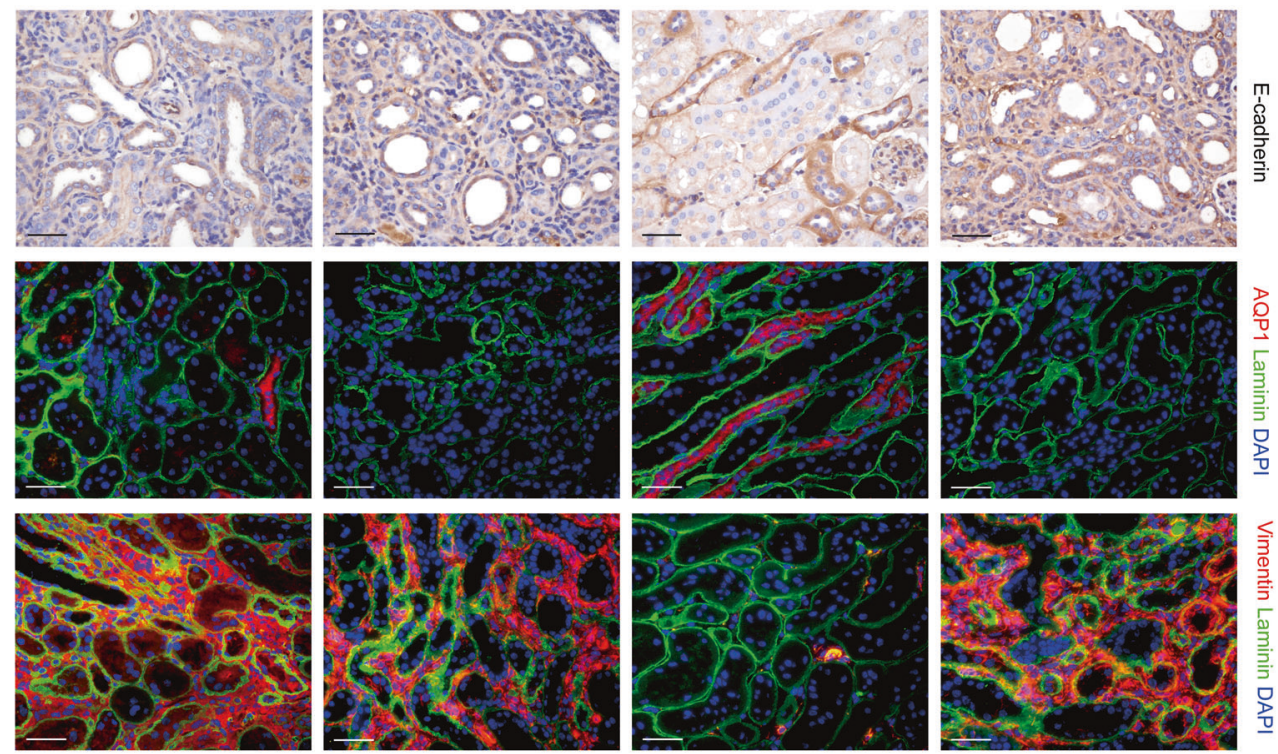

C

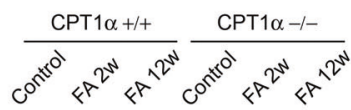

$170 \mathrm{kD}-\mathrm{ID} \rightarrow$ - Fibronectin

$130 \mathrm{kD}-\mathrm{B}=1$ - Collagen I
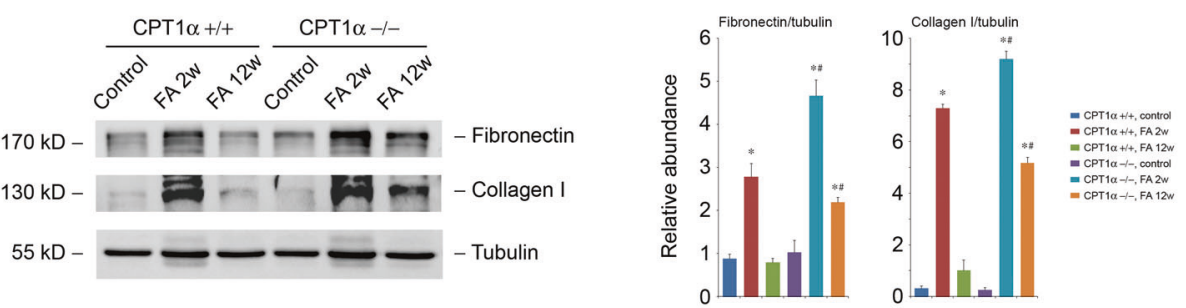

D

СРT $1 \alpha+/+$, FA $2 w$
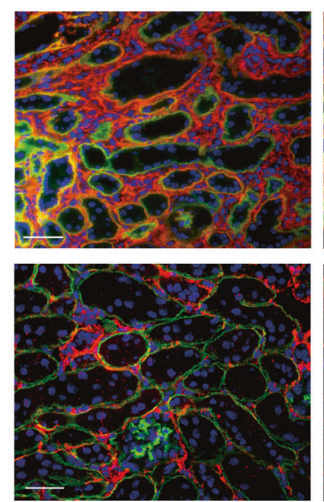

СРT $1 \alpha-/-$, FA $2 w$
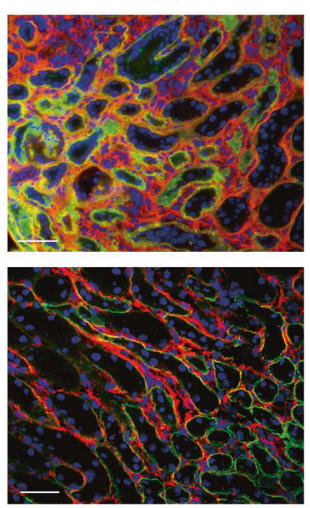

$\mathrm{CPT} 1 \alpha+/+$, FA $12 w$
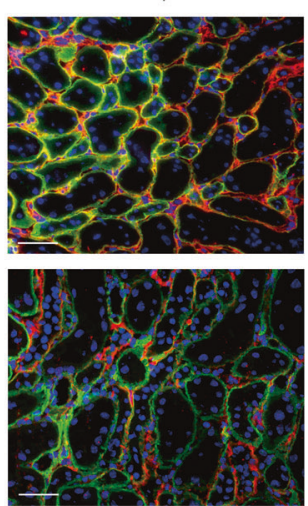

СРT $1 \alpha-/-$ FA $12 w$
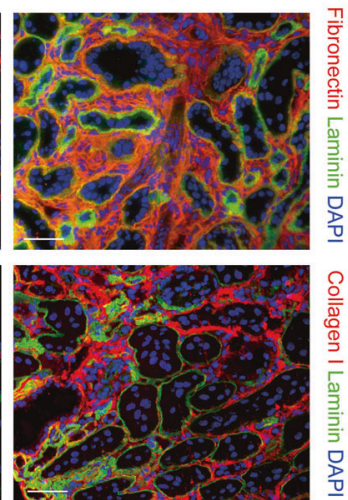

Fig. 4 CPT1 $\mathrm{a}$ is indispensable for preserve and recovery of tubular phenotype. A Western blot analysis of protein expression and graphic presentation of the relative abundance of E-cadherin, vimentin, and AQP-1 in kidney samples from groups as indicated. B Representative images of mice kidney samples immunostained with E-cadherin, AQP-1, and vimentin. C Western blot analysis of protein expression and graphic presentation of the relative abundance of fibronectin and collagen I in kidney samples from groups as indicated. D Representative images of mice kidney samples immunostained with fibronectin and collagen $\mathrm{I}$. ${ }^{*} P<0.05$ versus control. $\# P<0.05$ versus $C P T 1 \alpha+/+$. Scale bar, $50 \mu \mathrm{m}$. 
A
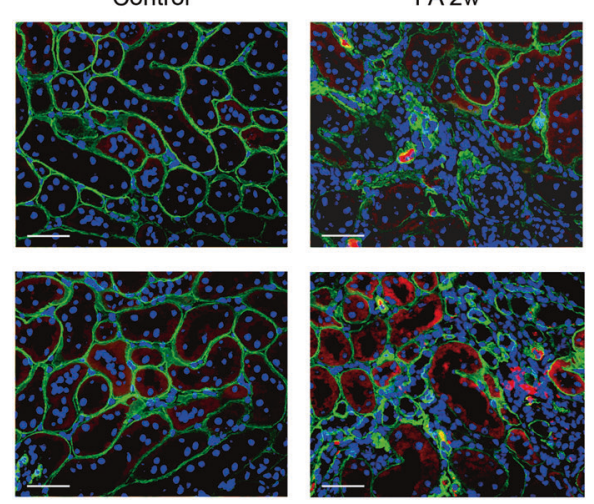

C
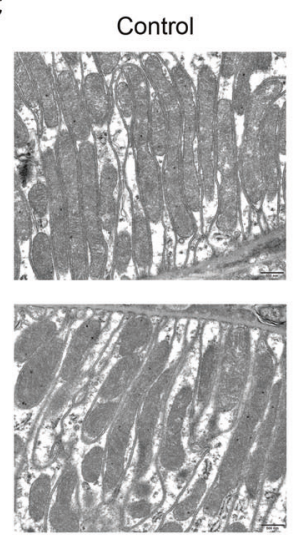

FA 2w
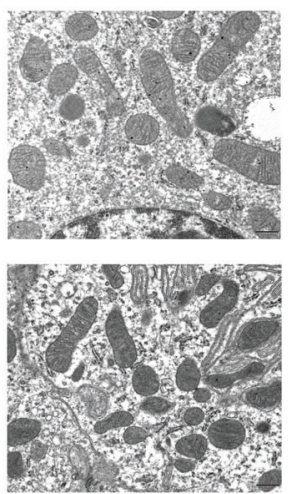

FA 12 w
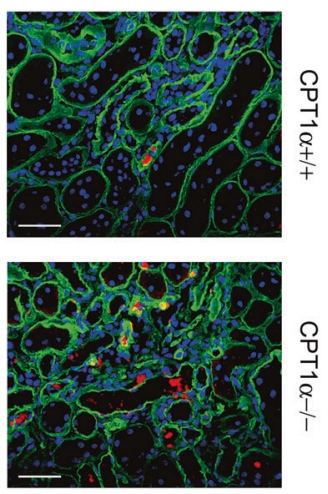

Bodipy Laminin DAPI

FA 12w
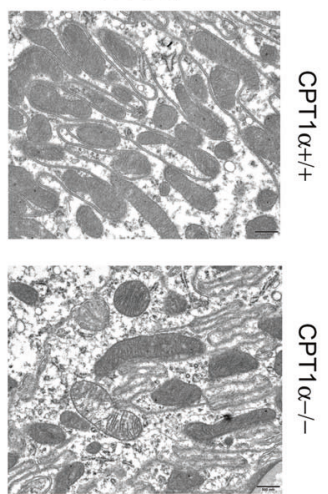

B

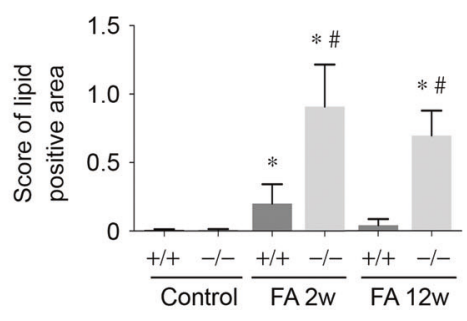

D

E
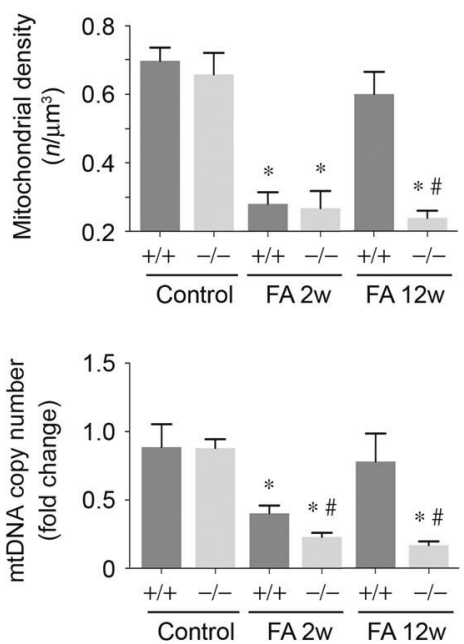

Fig. 5 CPT1a deficiency promotes lipid accumulation and mitochondrial mass reduction. A Representative images of kidney samples stained with bodipy. Red, bodipy. Green, laminin. Blue, nuclei. Scale bar, $50 \mu \mathrm{m}$. B Quantification of lipid positive area in kidney samples obtained from groups as indicated. $n=6$ for each group. C Representative TEM of the ultrastructure of mouse kidney tubular cells obtained from resin-embedded kidney sections from groups as indicated. Scale bars: $500 \mathrm{~nm}$. D Quantification of mean mitochondria per volume (n/ $\mu \mathrm{m}^{3}$ ) by morphometric analysis. $n=6$ for each group. $E$ mtDNA copy number was determined in kidneys from groups as indicated. Bar graphs represent the mean \pm SEM of fold changes. $n=6$ for each group. ${ }^{*} P<0.05$ versus control. $\# P<0.05$ versus CPT $1 \alpha+/+$.

conditions. Overexpression of CPT1a by transfection of specific plasmid markedly alleviated the reduction of baseline OCR, maximal respiration, spare respiration capacity, and ATP production (Fig. 8A, B) and resulted in reduced lipid accumulation (Fig. $8 C)$. Folic acid-induced suppression of tubular markers (E-cadherin and $A Q P 1)$ and promotion of vimentin and ECM markers (fibronectin and collagen I) were markedly relieved by upregulation of CPT1a compared to control (Fig. 8D, E).

\section{DISCUSSION}

In this study, we have identified CPT1a as a central molecule involved in the injury and repair response of damaged renal tubule cells. In vivo, tubular-specific ablation of CPT1a leads to sustained tubular damage and absence of the normal recovery of kidney function and dissipation of fibrosis after folic acid injury. In vitro, CPT1a promotes mitochondrial respiration and ATP production in cultured tubular epithelial cells. Moreover, we demonstrate that CPT1a relieves kidney injury and promotes tubular repair by preservation and recovery of tubular phenotype. Our results highlight the metabolism of proximal tubule cellular as one of the major drivers of cellular dedifferentiation and redifferentiation during kidney injury and repair.

The harmoniously coupling of energy metabolism and cellular state makes perfect sense in physiological conditions, especially in the energy-consuming proximal tubule. High glycolysis in effector T cells, while higher FAO exhibited in regulatory cells were the best-described example [27]. Dysregulated metabolism including increased glycolysis, OXPHOS, and fatty acid synthesis contributes to autoimmune diseases [28-30]. The remarkable effect of sodium-glucose cotransporter 2 inhibitors in relieving the deterioration of renal function may possibly attribute to modulating tubular energy metabolism [31, 32]. It is recently reported that proximal tubular cell differentiation is altered in kidney disease, which shows the strongest and most reproducible association with metabolism, especially FAO and OXPHOS [14]. In this study, we established the role of CPT1a, the rate-limiting enzyme of FAO in the coupling of tubular cell phenotype and the metabolism.

CPT1 enzyme has three isoforms with tissue-specific expression and encoded by a different gene. The liver and kidney CPT1a is expressed by CPT1A gene, while the skeletal and cardiac muscle by the CPT1B gene, and the brain by the CPT1C gene [33]. Although increasing evidence has demonstrated an association between the decreased expression of CPT1a and kidney diseases development [6], more direct evidence is still lacking. We, therefore, breed tetO-CPT1a mice with Pax8rt-TA mice and feed them with a doxycycline-containing diet to generate the inducible tubular-specific CPT1a deletion in adult mice and therefore avoid the embryo and pre-mature lethality. CPT1 deficiency in clinical settings is an autosomal-recessively inherited condition mainly affecting liver, heart, muscle, and kidney. Half a century ago, a case 
A

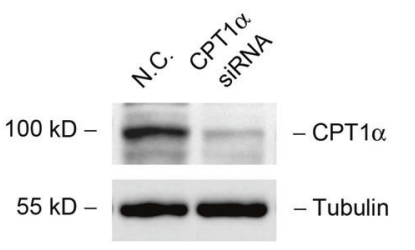

B

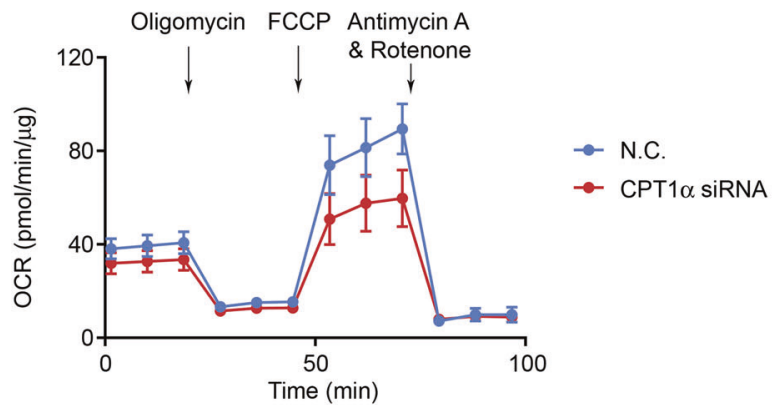

C
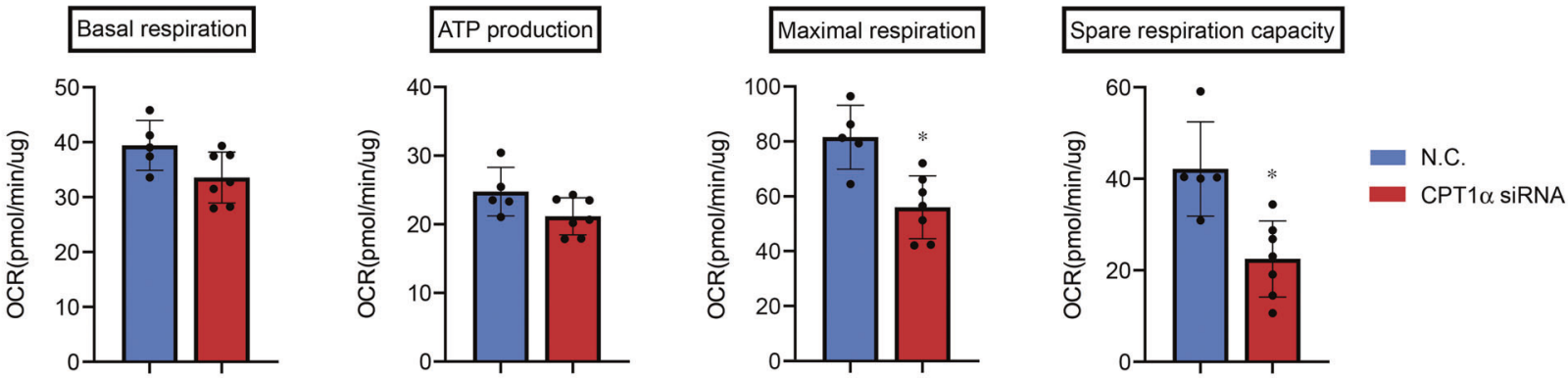

D

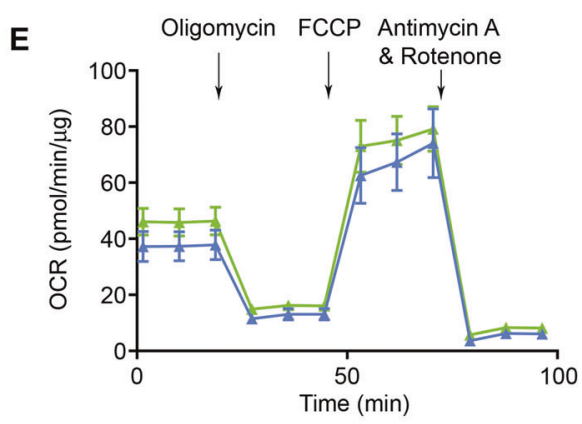

$$
\begin{aligned}
& \star \text { pcDNA3 } \\
& \star \text { pCPT1 } \alpha
\end{aligned}
$$

F
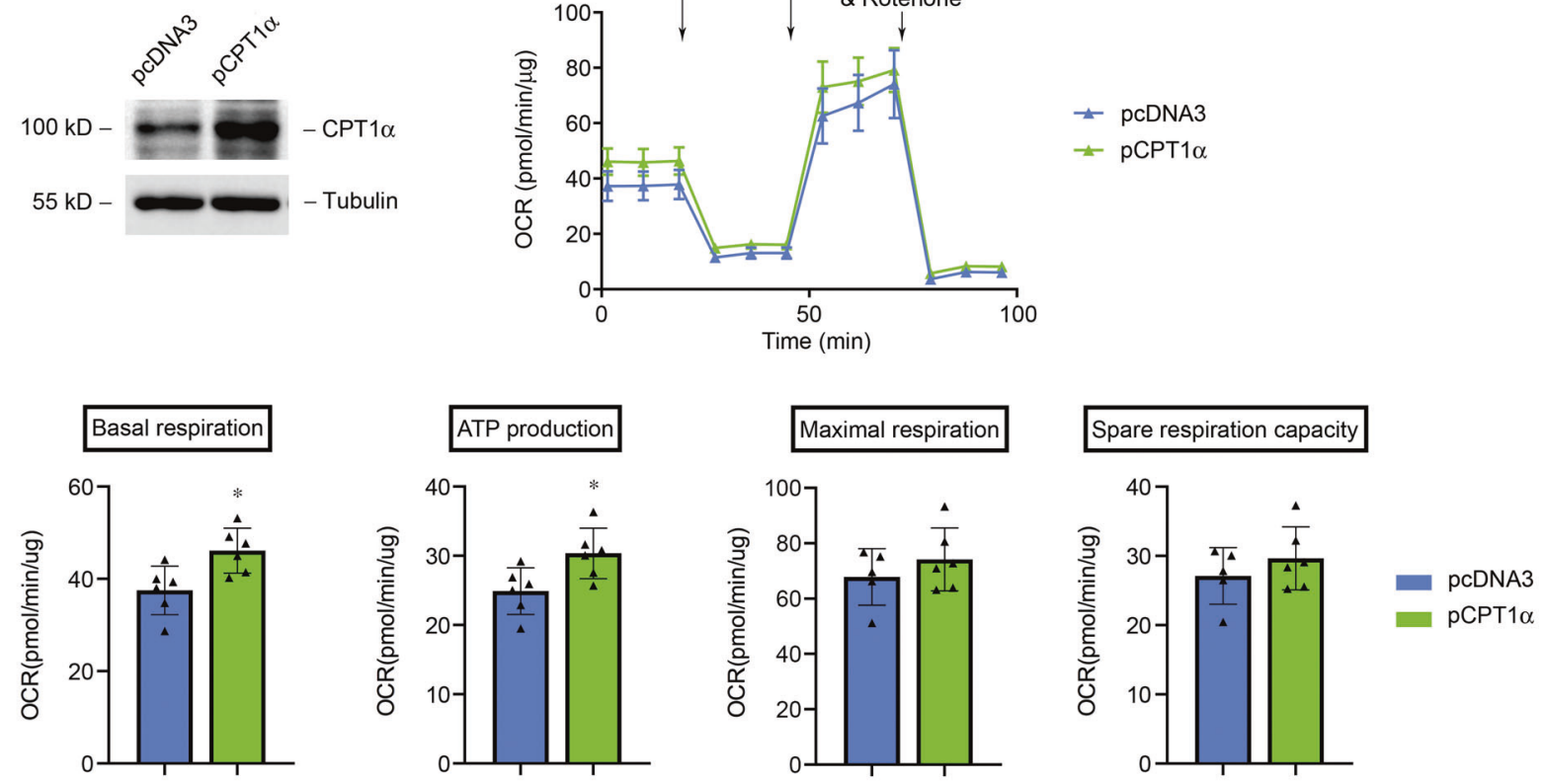

Fig. 6 CPT1a regulates respiration and ATP production in cultured tubular epithelial cells. A Western blot analysis of protein expression of CPT1 $\alpha$ in cultured tubular cells after transfection with N.C. or CPT1 $\alpha$ siRNA. B Representative traces show OCR in tubular cells. C Summary OCR data from 5 to 6 independent experiments are shown. ${ }^{*} P<0.05$ versus N.C. D Western blot analysis of protein expression of CPT1 $\alpha$ in cultured tubular cells after transfection with pCDNA3 or CPT1 $\alpha$ plasmid. E Representative traces show OCR in tubular cells. F Summary OCR data analyzed from 5 to 6 independent experiments are shown. ${ }^{*} P<0.05$ versus pcDNA3. One $\mu \mathrm{mol} / \mathrm{L} \mathrm{Oligomycin,} 0.75 \mu \mathrm{mol} / \mathrm{L}$ of FCCP, $1 \mu \mathrm{mol} / \mathrm{L}$ of antimycin $A$ and rotenone were added where indicated.

of CPT1 deficiency was reported with clinical manifestation of kidney involvement, including pigmenturia and rhabdomyolysis [34]. However, the symptoms are intermittent and induced by certain stress, and the stimulus that triggers clinical presentations differ between each patient $[35,36]$. Not surprisingly, we found no significant difference in the phenotype of knockout (CPT1a-/-) and their littermates $(\mathrm{CPT} 1 \mathrm{a}+/+)$ at the time before the folic acid injury. Although the basal OCR tends to decreased in CPT1a deficient PTECs, the tubular phenotype and lipid remain normal. Whether there is a compensatory mechanism for FAO in the absence of CPT1a in normal conditions needs further investigations.

Of note, the severity of acute kidney injury induced by folic acid was comparable between CPT1a+/+ and CPT1a-/- mice (data not shown). It was reported that metabolic switch from FAO toward glycolysis that occurred early after acute injury was indispensable for tubular regeneration and recovery [9]. This even coincides with a previous study suggesting inhibition of the CPT enzyme to protect PTECs during hypoxia [37]. We postulated that PTECs may become less dependent on FAO 
A

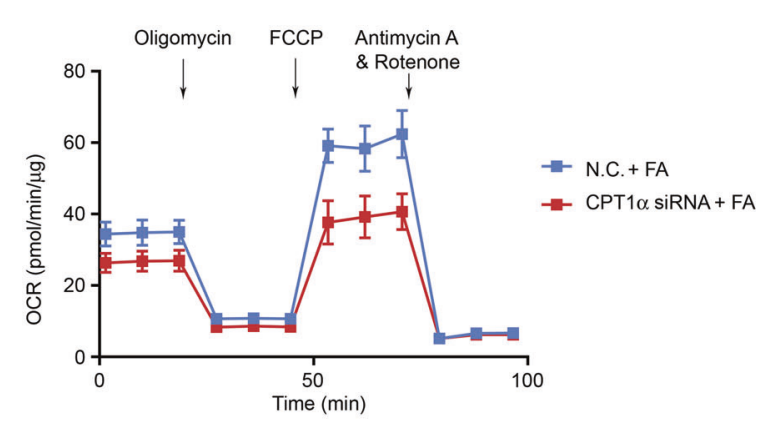

C

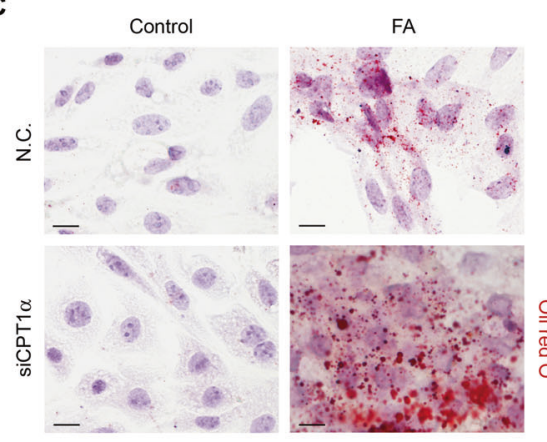

D

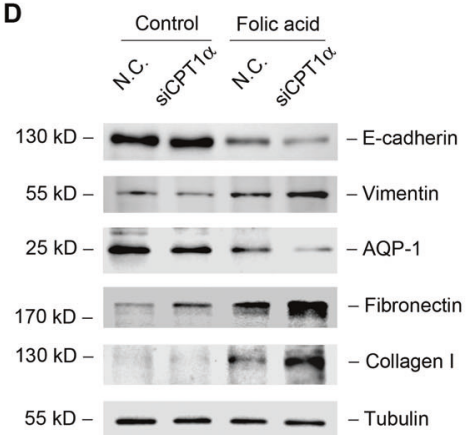

Basal respiration
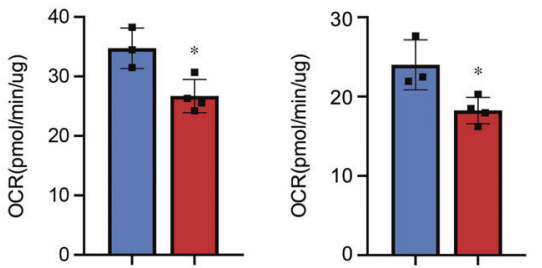

N.C. + FA

CPT1 $\alpha$ siRNA+FA
Maximal respiration

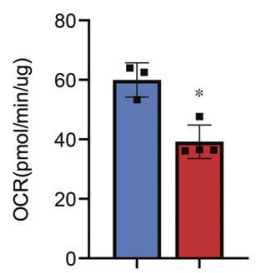

E

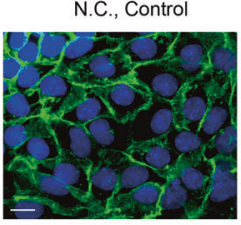

siCPT1 $\alpha$, Control
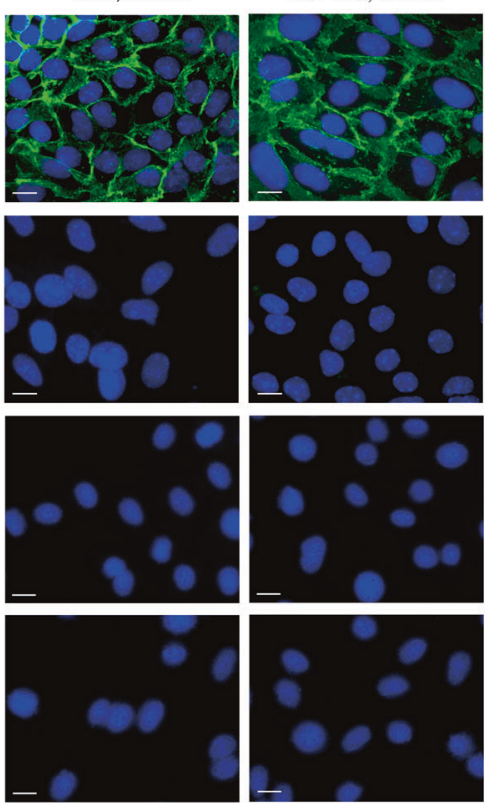
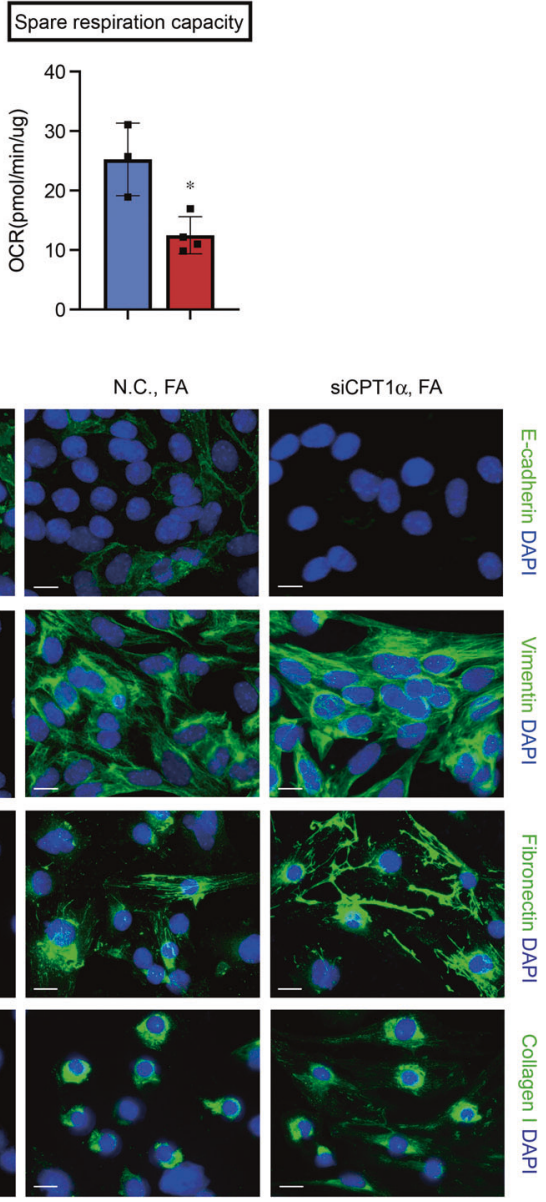

Fig. 7 Tubular cells with reduced CPT1a are more sensitive to folic acid-induced alternation. A Representative traces show OCR in folic acid-treated tubular cells after transfection with N.C. or CPT1 $\alpha$ siRNA. Oligomycin, FCCP, antimycin A, and rotenone were added where indicated. B Summary OCR data from 3 to 4 independent experiments are shown. ${ }^{*} P<0.05$ versus N.C. + FA. C Representative images of tubular cells exposed to treatments as indicated stained with oil red O. D Western blot analysis of protein expression of E-cadherin, vimentin, AQP-1, fibronectin, and collagen I in tubular cells. E Representative images of tubular cells immunostained with E-cadherin, vimentin, fibronectin, and collagen I. Scale bar, $10 \mu \mathrm{m}$.

in the acute phase after injury. However, the irreversibility of the metabolic switch characterizes persistent dedifferentiation of PTECs and results in severe renal fibrosis in the late phase [9].

As the rate-limiting enzyme for medium and long fatty acid shuttling into mitochondria, ablation of CPT1 a certainly hampered the recovery of preferred FAO in PTECs and results in more severe fatty acid deposition and tubulointerstitial fibrosis. Verónica Miguel found a correlation between short-/medium-acylcarnitine levels and renal function [18]. Afshinnia et al. found impaired fatty acid $\beta$-oxidation in severe kidney disease, but they did not check the CPT1a levels [38]. In patients with diabetes, kidney involvement is associated with incomplete FAO and alternations of related enzymes including CPT1a [6]. Here, the recovery of FAO, accompanied with CPT1 most likely contributes to kidney repair; however, as CPT1a is rate-limiting for FAO in the mitochondria, and overexpression of CPT1a mitigate FAO impairment [18], a clear distinction between the roles of CPT1a and FAO in kidney injury and repair remains obscure.

Mitochondria are the most important intracellular organelles for ATP production. The active reabsorption of large quantities of solutes in kidney tubule needs high energy that relies on mitochondrial oxidation. As the greatest reabsorption section, the proximal tubules primarily apply aerobic respiration for ATP production [2] and equipped with abundant of mitochondria. Quality control and homeostasis of mitochondria are indispensable for the maintenance of a variety of cellular processes in normal kidney. It is conceivable that mitochondria are closely associated with kidney injury and repair. After the initial insult, mitochondrial mass reduction together with the metabolic 
A

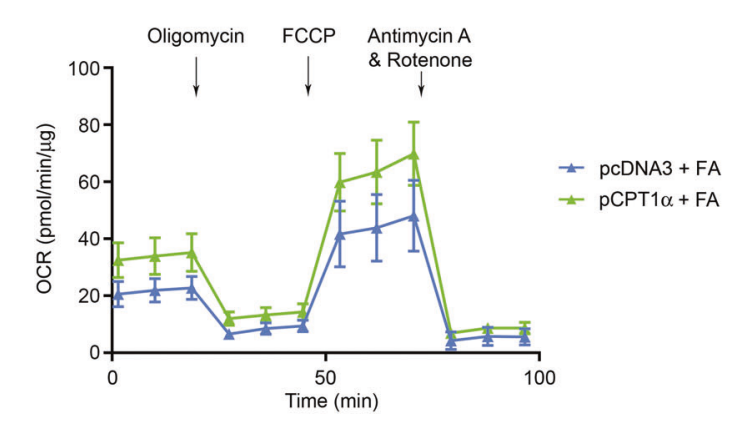

C

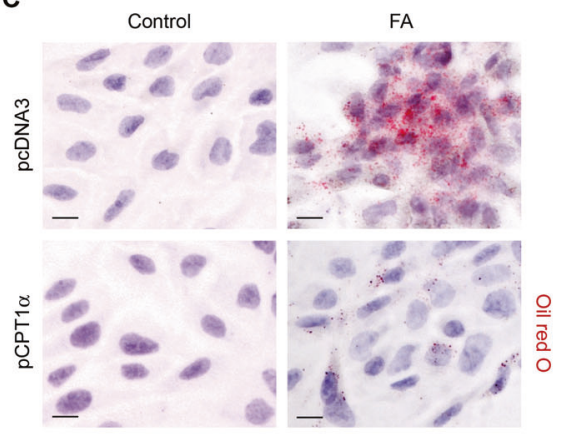

D

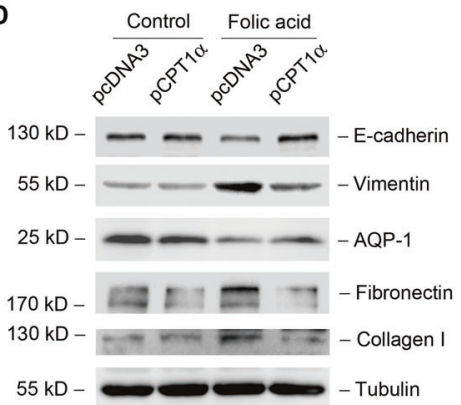

$\mathbf{E}$
B

Basal respiration $\quad$ ATP production
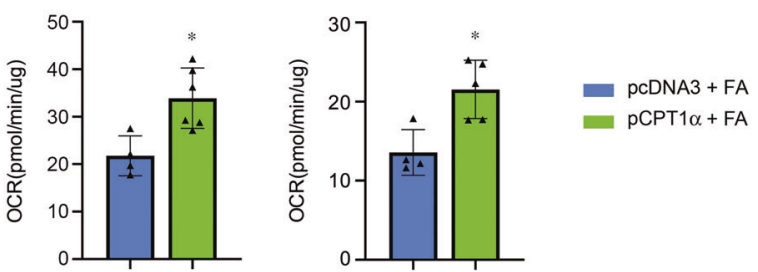

Maximal respiration

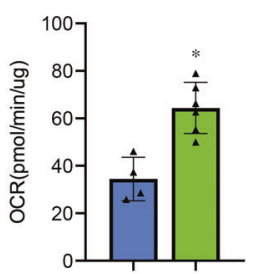

Spare respiration capacity
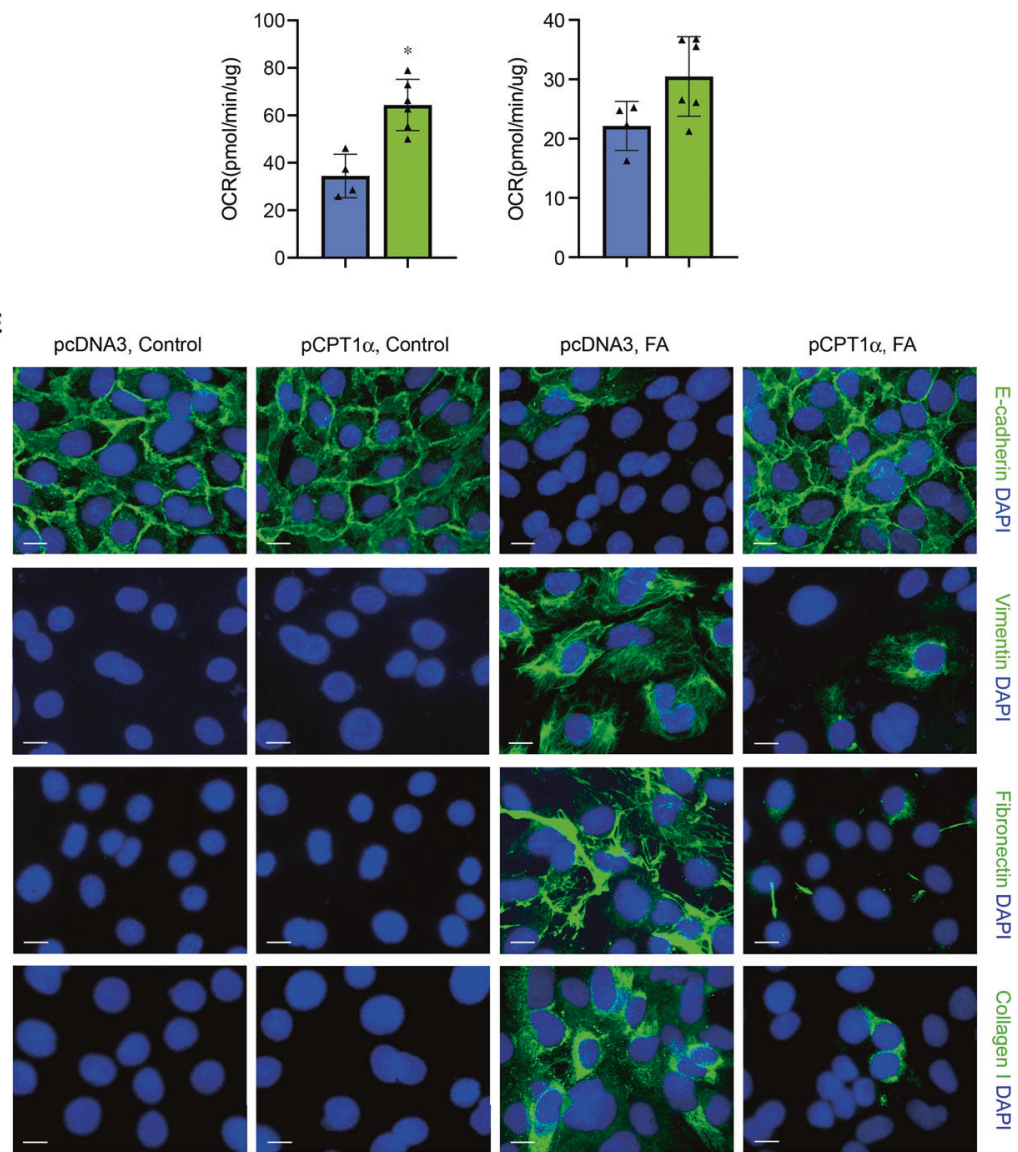

Fig. 8 CPT1a protects mitochondrial respiration and prevents against folic acid-induced tubular cell damage. A Representative traces show OCR in folic acid-treated tubular cells after transfection with pCDNA3 or CPT1 $\alpha$ plasmid. Oligomycin, FCCP, antimycin A, and rotenone were added. B Summary OCR data from 3 to 4 independent experiments are shown. ${ }^{*} P<0.05$ versus pCDNA3+FA. C Representative images of tubular cells exposed to treatments as indicated stained with oil red O. D Western blot analysis of protein expression of E-cadherin, vimentin, AQP-1, fibronectin, and collagen I in tubular cells. E Representative images of tubular cells immunostained with E-cadherin, vimentin, fibronectin, and collagen I. Scale bar, $10 \mu \mathrm{m}$.

transfer from OXPHOS to anaerobic glycolysis observed in kidney tubules led to dedifferentiation and proliferation of tubular cells [9]. Reversal of the reduction promotes normal repairment of tubules. However, persist and progressive damage results in failure of tubular redifferentiation, suggesting a key role of mitochondrial regression during the repair. A recent study has suggested that overexpression of CPT1a protects from kidney fibrosis by restoring mitochondrial homeostasis [18]. Mitochondria regulate cell differentiation mainly dependent on the control of energy metabolism. Surviving tubular cells undergo a series of continuous alternations to repair injured kidney tubules, including dedifferentiation, proliferation, migration, and finally redifferentiation into mature tubular cells. Increasing evidence suggests that mitochondrial dysfunction contributes critically to the pathogenesis of injury and incomplete kidney repair. Mitochondrial protection before the injury is protective [39], while mitochondrial protection after injury mitigates the progression to tubular atrophy and chronic fibrosis [40].

In summary, we showed the vital role of metabolism in driving PTECs phenotype state. CPT1a couples cell metabolism and differentiation state by regulating mitochondrial respiration. The work provides new opportunities to manipulate proximal tubular cell differentiation, metabolism, and kidney tissue fate based on the reliance of tubular function on energy metabolic molecules such as CPT1a.

\section{REFERENCES}

1. Bhargava $P$, Schnellmann RG. Mitochondrial energetics in the kidney. Nat Rev Nephrol. 2017;13:629-46. 
2. Forbes JM, Thorburn DR. Mitochondrial dysfunction in diabetic kidney disease. Nat Rev Nephrol. 2018;14:291-312.

3. Forbes JM. Mitochondria-power players in kidney function? Trends Endocrinol Metab. 2016;27:441-2.

4. Rastaldi MP, Ferrario F, Giardino L, Dell'Antonio G, Grillo C, Grillo P, et al. Epithelial-mesenchymal transition of tubular epithelial cells in human renal biopsies. Kidney Int. 2002;62:137-46.

5. Yang J, Liu Y. Dissection of key events in tubular epithelial to myofibroblast transition and its implications in renal interstitial fibrosis. Am J Pathol. 2001;159:1465-75.

6. Kang HM, Ahn SH, Choi P, Ko YA, Han SH, Chinga F, et al. Defective fatty acid oxidation in renal tubular epithelial cells has a key role in kidney fibrosis development. Nat Med. 2015;21:37-46.

7. Bonventre JV, Weinberg JM. Recent advances in the pathophysiology of ischemic acute renal failure. J Am Soc Nephrol. 2003;14:2199-210.

8. Chang-Panesso M, Humphreys BD. Cellular plasticity in kidney injury and repair. Nat Rev Nephrol. 2017;13:39-46.

9. Lan $R$, Geng $H$, Singha PK, Saikumar $P$, Bottinger EP, Weinberg JM, et al. Mitochondrial pathology and glycolytic shift during proximal tubule atrophy after ischemic AKI. J Am Soc Nephrol. 2016;27:3356-67.

10. James MT, Bhatt M, Pannu N, Tonelli M. Long-term outcomes of acute kidney injury and strategies for improved care. Nat Rev Nephrol. 2020;16:193-205.

11. Cao H, Luo J, Zhang $Y$, Mao X, Wen P, Ding H, et al. Tuberous sclerosis 1 (Tsc1) mediated mTORC1 activation promotes glycolysis in tubular epithelial cells in kidney fibrosis. Kidney Int. 2020;98:686-98.

12. Shen $Y$, Jiang $L$, Wen $P, Y e ~ Y$, Zhang $Y$, Ding $H$, et al. Tubule-derived lactate is required for fibroblast activation in acute kidney injury. Am J Physiol Ren Physiol. 2020;318:F689-F701.

13. Ding $H$, Jiang $L, X u$ J, Bai $F$, Zhou $Y$, Yuan Q, et al. Inhibiting aerobic glycolysis suppresses renal interstitial fibroblast activation and renal fibrosis. Am J Physiol Ren Physiol. 2017;313:F561-F75.

14. Dhillon P, Park J, Hurtado Del Pozo C, Li L, Doke T, Huang S, et al. The nuclear receptor ESRRA protects from kidney disease by coupling metabolism and differentiation. Cell Metab. 2020;33:379-394.e8.

15. Idrovo JP, Yang WL, Nicastro J, Coppa GF, Wang P. Stimulation of carnitine palmitoyltransferase 1 improves renal function and attenuates tissue damage after ischemia/reperfusion. J Surg Res. 2012;177:157-64.

16. Idrovo JP, Yang WL, Matsuda A, Nicastro J, Coppa GF, Wang P. Post-treatment with the combination of 5-aminoimidazole-4-carboxyamide ribonucleoside and carnitine improves renal function after ischemia/reperfusion injury. Shock 2012;37:39-46.

17. Price NL, Miguel V, Ding W, Singh AK, Malik S, Rotllan N, et al. Genetic deficiency or pharmacological inhibition of miR-33 protects from kidney fibrosis. JCI Insight. 2019;4:e131102.

18. Miguel V, Tituana J, Herrero Jl, Herrero L, Serra D, Cuevas P, et al. Renal tubule Cpt1a overexpression protects from kidney fibrosis by restoring mitochondrial homeostasis. J Clin Invest. 2021;131:e140695.

19. Yang HC, Zuo Y, Fogo AB. Models of chronic kidney disease. Drug Disco Today Dis Models. 2010;7:13-9.

20. Terryn S, Jouret F, Vandenabeele F, Smolders I, Moreels $M$, Devuyst $O$, et al. A primary culture of mouse proximal tubular cells, established on collagen-coated membranes. Am J Physiol Ren Physiol. 2007;293:F476-85.

21. Ke Q, Yuan Q, Qin N, Shi C, Luo J, Fang Y, et al. UCP2-induced hypoxia promotes lipid accumulation and tubulointerstitial fibrosis during ischemic kidney injury. Cell Death Dis. 2020;11:26.

22. Lee M, Katerelos M, Gleich K, Galic S, Kemp BE, Mount PF, et al. Phosphorylation of acetyl-CoA carboxylase by AMPK reduces renal fibrosis and is essential for the anti-fibrotic effect of metformin. J Am Soc Nephrol. 2018;29:2326-36.

23. Santos S, Bosch RJ, Ortega A, Largo R, Fernandez-Agullo T, Gazapo R, et al. Upregulation of parathyroid hormone-related protein in folic acid-induced acute renal failure. Kidney Int. 2001;60:982-95.

24. Qin N, Cai T, Ke Q, Yuan Q, Luo J, Mao X, et al. UCP2-dependent improvement of mitochondrial dynamics protects against acute kidney injury. J Pathol. 2019;247:392-405.

25. Cai T, Ke Q, Fang Y, Wen $P$, Chen H, Yuan Q, et al. Sodium-glucose cotransporter 2 inhibition suppresses HIF-1alpha-mediated metabolic switch from lipid oxidation to glycolysis in kidney tubule cells of diabetic mice. Cell Death Dis. 2020;11:390.

26. Lim BJ, Yang HC, Fogo AB. Animal models of regression/progression of kidney disease. Drug Disco Today Dis Models. 2014;11:45-51.

27. Angelin A, Gil-de-Gomez L, Dahiya S, Jiao J, Guo L, Levine MH, et al. Foxp3 reprograms $\mathrm{T}$ cell metabolism to function in low-glucose, high-lactate environments. Cell Metab. 2017;25:1282-93. e7

28. Kornberg MD, Bhargava P, Kim PM, Putluri V, Snowman AM, Putluri N, et al. Dimethyl fumarate targets GAPDH and aerobic glycolysis to modulate immunity. Science 2018;360:449-53.
29. Shen Y, Wen Z, Li Y, Matteson EL, Hong J, Goronzy JJ, et al. Metabolic control of the scaffold protein TKS5 in tissue-invasive, proinflammatory T cells. Nat Immunol. 2017;18:1025-34.

30. Yin Y, Choi SC, Xu Z, Perry DJ, Seay $H$, Croker BP, et al. Normalization of CD4+ T cell metabolism reverses lupus. Sci Transl Med. 2015;7:274ra18.

31. Heerspink HJL, Stefansson BV, Correa-Rotter R, Chertow GM, Greene T, Hou FF, et al. Dapagliflozin in patients with chronic kidney disease. N Engl J Med. 2020;383:1436-46.

32. Hesp AC, Schaub JA, Prasad PV, Vallon V, Laverman GD, Bjornstad P, et al. The role of renal hypoxia in the pathogenesis of diabetic kidney disease: a promising target for newer renoprotective agents including SGLT2 inhibitors? Kidney Int 2020;98:579-89.

33. Saggerson ED, Carpenter CA. Carnitine palmitoyltransferase and carnitine octanoyltransferase activities in liver, kidney cortex, adipocyte, lactating mammary gland, skeletal muscle, and heart. FEBS Lett. 1981;129:229-32.

34. Brownell AK, Severson DL, Thompson CD, Fletcher T. Cold induced rhabdomyolysis in carnitine palmyityl transferase deficiency. Can J Neurol Sci. 1979;6:367-70.

35. Di Donato S, Castiglione A, Rimoldi M, Cornelio F, Vendemia F, Cardace G, et al Heterogeneity of carnitine-palmitoyltransferase deficiency. J Neurol Sci. 1981;50:207-15.

36. Falik-Borenstein ZC, Jordan SC, Saudubray JM, Brivet M, Demaugre F, Edmond J, et al. Brief report: renal tubular acidosis in carnitine palmitoyltransferase type 1 deficiency. N Engl J Med. 1992;327:24-7.

37. Portilla D. Carnitine palmitoyl-transferase enzyme inhibition protects proximal tubules during hypoxia. Kidney Int. 1997;52:429-37.

38. Afshinnia F, Rajendiran TM, Soni T, Byun J, Wernisch S, Sas KM, et al. Impaired beta-oxidation and altered complex lipid fatty acid partitioning with advancing CKD. J Am Soc Nephrol. 2018;29:295-306.

39. Perry HM, Huang L, Wilson RJ, Bajwa A, Sesaki H, Yan Z, et al. Dynamin-related protein 1 deficiency promotes recovery from AKI. J Am Soc Nephrol. 2018;29:194-206.

40. Szeto HH, Liu S, Soong Y, Seshan SV, Cohen-Gould L, Manichev V, et al. Mitochondria protection after acute ischemia prevents prolonged upregulation of IL1 beta and IL-18 and arrests CKD. J Am Soc Nephrol. 2017;28:1437-49.

\section{ACKNOWLEDGEMENTS}

We thank Prof. Donghai Wu from Key Laboratory of Regenerative Biology, Guangzhou Institute of Biomedicine and Health, Chinese Academy of Sciences for kindly providing us the CPT1 $a^{\text {flox/flox }}$ mice.

\section{AUTHOR CONTRIBUTIONS}

Y.Z. and J.Y. conceived and designed the study; Q.Y., Y.L., H.D., Q.K., C.S., J.L., and L.J. carried out experiments; Y.Z., J.Y., and L.J. analyzed the data; Y.Z. and Q.Y. made the figures; Y.Z. drafted and J.Y. revised the paper; all authors approved the final version of the paper.

\section{FUNDING}

The work was supported by the Natural Science Foundation of Jiangsu province: General program BK20201497 to Yang Zhou, National Natural Science Foundation of China: General program 81873618 to Junwei Yang. These funders had no role in study design, data collection, and analysis, preparation of the paper, or decision to publish.

\section{ETHICS}

The study protocol was approved by the Ethical Commission in Nanjing Medical University.

\section{COMPETING INTERESTS}

The authors declare no competing interests.

\section{ADDITIONAL INFORMATION}

Correspondence and requests for materials should be addressed to L.J., J.Y. or Y.Z

Reprints and permission information is available at http://www.nature.com/ reprints

Publisher's note Springer Nature remains neutral with regard to jurisdictional claims in published maps and institutional affiliations. 
(c) (i) Open Access This article is licensed under a Creative Commons Attribution 4.0 International License, which permits use, sharing, adaptation, distribution and reproduction in any medium or format, as long as you give appropriate credit to the original author(s) and the source, provide a link to the Creative Commons license, and indicate if changes were made. The images or other third party material in this article are included in the article's Creative Commons license, unless indicated otherwise in a credit line to the material. If material is not included in the article's Creative Commons license and your intended use is not permitted by statutory regulation or exceeds the permitted use, you will need to obtain permission directly from the copyright holder. To view a copy of this license, visit http://creativecommons. org/licenses/by/4.0/.

(c) The Author(s) 2021 